\title{
A computational system for lattice QCD with overlap Dirac quarks*
}

\author{
Ting-Wai Chiu ${ }^{a}$, Tung-Han Hsieh ${ }^{a}$, Chao-Hsi Huang ${ }^{a}$, Tsung-Ren Huang ${ }^{a}$

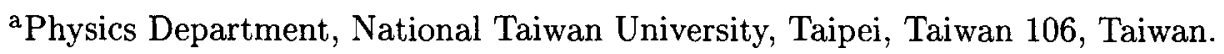

We outline the essential features of a Linux PC cluster which is now being developed at National Taiwan University, and discuss how to optimize its hardware and software for lattice QCD with overlap Dirac quarks. At present, the cluster constitutes of 30 nodes, with each node consisting of one Pentium 4 processor (1.6/2.0 $\mathrm{GHz}$ ), one Gbyte of PC800 RDRAM, one 40/80 Gbyte hard disk, and a network card. The speed of this system is estimated to be $30 \mathrm{Gflops,} \mathrm{and} \mathrm{its} \mathrm{price/performance} \mathrm{ratio} \mathrm{is} \mathrm{better} \mathrm{than} \$ 1.0 /$ Mflops for 64 -bit (double precision) computations in quenched lattice QCD with overlap Dirac quarks.

\section{Introduction}

It is well known that extracting physics from lattice $\mathrm{QCD}$ requires computing power exceeding that of any desktop personal computer currently available in the market. Therefore, for one without supercomputer resources, building a computational system seems to be inevitable if one really wishes to pursue a meaningful number of any physical quantity from lattice QCD. However, the feasibility of such a project depends not only on the funding, but also on the theoretical advancement of the subject, namely, the realization of exact chirally symmetry on the lattice $[1,2]$. Now, if we also take into account of the current price/performance of $\mathrm{PC}$ hardware components, it seems to be the right timing to rejuvenate the project [3] with a new goal - to build a computational system for lattice QCD with exact chiral symmetry. In this paper, we outline the essential features of a Linux PC cluster which is now being developed at National Taiwan University, and discuss how to optimize its hardware and software for lattice QCD with overlap Dirac quarks. More detailed descriptions have been given in Ref. [4].

First, we start from quenched QCD, and compute quark propagators in the gluon field background, for a sequence of configurations generated stochastically with weight $\exp \left(-\mathcal{A}_{g}\right)$. Then the hardronic observables such as meson and

\footnotetext{
*This work was supported in part by National Science Council R.O.C. under the grant number NSC90-2112M002-021
}

baryon correlation functions can be constructed, and from which the hadron masses and decay constants can be extracted. In general, one requires that any quark propagator coupling to physical hadrons must be of the form [5]

$$
\left(D_{c}+m_{q}\right)^{-1} \text {, }
$$

where $m_{q}$ is the bare quark mass, and $D_{c}$ is a chirally symmetric and anti-hermitian Dirac operator $^{2}$. For any massless lattice Dirac operator $D$ satisfying the Ginsparg-Wilson relation [6]

$D \gamma_{5}+\gamma_{5} D=2 r a D \gamma_{5} D$

it can be written as $D=D_{c}\left(1+r a D_{c}\right)^{-1}[7]$, and the bare quark mass is naturally added to the $D_{c}$ in the numerator [5],

$D\left(m_{q}\right)=\left(D_{c}+m_{q}\right)\left(1+r a D_{c}\right)^{-1}$.

Then the quenched quark propagator becomes

$$
\left(D_{c}+m_{q}\right)^{-1}=\left(1-r m_{q} a\right)^{-1}\left[D\left(m_{q}\right)^{-1}-r a\right] .
$$

If we fix one of the end points at $(\overrightarrow{0}, 0)$ and use the Hermitcity $D^{\dagger}=\gamma_{5} D \gamma_{5}$, then only 12 (3 colors times 4 Dirac indices) columns of

$$
D\left(m_{q}\right)^{-1}=D^{\dagger}\left(m_{q}\right)\left\{D\left(m_{q}\right) D^{\dagger}\left(m_{q}\right)\right\}^{-1}
$$

are needed for computing the time correlation functions of hadrons. Now our problem is how

\footnotetext{
${ }^{2}$ Here we assume that $D_{c}$ is doubler-free, and has correct continuum behavior, and $D=D_{c}\left(1+r a D_{c}\right)^{-1}$ is exponentially local for a range of $r>0$.
} 
to optimize a PC cluster to compute $D\left(m_{q}\right)^{-1}$ for a set of bare quark masses.

For overlap Dirac quarks, we need to solve the following linear system by (multi-mass) conjugate gradient $(\mathrm{CG})$,

$D D^{\dagger} Y=\left(\alpha+\eta P_{ \pm} H_{w} \frac{1}{\sqrt{H_{w}^{2}}}\right) Y=\mathbf{I}$

where $P_{ \pm}=\left(\gamma_{5} \pm 1\right) / 2, \alpha=2 m_{0}^{2}+m_{q}^{2} / 2$, $\eta=2 m_{0}^{2}-m_{q}^{2} / 2$, and $m_{0}$ is fixed to 1.3 in our computations. Then the quark propagators can be obtained via (3). With Zolotarev optimal rational approximation $[8,9]$ to $\left(H_{w}^{2}\right)^{-1 / 2}$, the multiplication $\left(h_{w} \equiv H_{w} / \lambda_{\min }\right)$

$H_{w} \frac{1}{\sqrt{H_{w}^{2}}} Y \simeq h_{w}\left(h_{w}^{2}+c_{2 n}\right) \sum_{l=1}^{n} \frac{b_{l}}{h_{w}^{2}+c_{2 l-1}} Y$

can be evaluated by invoking another (multimass) conjugate gradient process to the lincar systems

$\left(h_{w}^{2}+c_{2 l-1}\right) Z_{l}=Y, \quad l=1, \cdots, n$.

where the coefficients $c_{l}, b_{l}$ and $d_{0}$ have been given explicitly in Ref. [10].

In order to improve the accuracy of the rational approximation as well as to reduce the number of iterations in the inner CG loop, it is crucial to project out the largest and some low-lying eigenmodes of $H_{w}^{2}$. Denoting these eigenmodes by

$H_{w} u_{j}=\lambda_{j} u_{j}, \quad j=1, \cdots, k$,

then we project the linear systems (6) to the complement of the vector space spanned by these eigenmodes

$\left(h_{w}^{2}+c_{2 l-1}\right) \bar{Z}_{l}=\bar{Y} \equiv\left(1-\sum_{j=1}^{k} u_{j} u_{j}^{\dagger}\right) Y$.

Thus the r.h.s. of (5) can be rewritten as

$h_{w}\left(h_{w}^{2}+c_{2 n}\right) \sum_{l=1}^{n} b_{l} \bar{Z}_{l}+\sum_{j=1}^{k} \frac{\lambda_{j}}{\sqrt{\lambda_{j}^{2}}} u_{j} u_{j}^{\dagger} Y \equiv S$

Then the breaking of exact chiral symmetry (2) can be measured in terms of

$\sigma=\frac{\left|S^{\dagger} S-Y^{\dagger} Y\right|}{Y^{\dagger} Y}$.
In practice, one has no difficulties to attain $\sigma<$ $10^{-12}$ for most gauge configurations on a finite lattice (Table 1).

Now the computation of overlap Dirac quark propagator involves two nested conjugate gradient loops: the so-called inner CG loop (8), and the outer CG loop (4).

\section{Optimization}

Next we address the question how to configure the hardware and software of a PC cluster such that it can attain the optimal price/performance ratio for the execution of the nested CG loops.

The vital observation is that not all column vectors are used simultaneously at any step of the nested CG loops, and also the computationally intense part is at the inner CG loop (8). Thus we can use the hard disk as virtual memory for the storage of the intermediate solution vectors and their conjugate gradient vectors at each iteration of the outer CG loop, while the CPU is working on the inner CG loop. Then the amount of required memory at each node can be greatly reduced. It is easy to figure out [4] the minimum memory required for accommodating the link variables and all relevant vectors for the inner CG loop,

$N_{c g}^{\text {min }}=384 \times N_{s}(n+3)$ byte,

where $n$ is the degree of the Zolotarev rational polynomial, and $N_{s}$ is the total number of lattice sites. For computations of quark propagators on the $16^{3} \times 32$ lattice with $n=16$, (11) gives $N_{c g}^{m i n}=0.912$ Gbyte, which can be implemented in a single node with one Gbyte of memory (i.e., four stripes of 256 Mbyte memory modules). Then the maximum speed of the cluster can be attained since there is no communication overheads. Moreover, the time for disk $\mathrm{I} / \mathrm{O}$ (at the interface of inner and outer CG loops) only constitutes a few percent of the total time for the entire nested CG loops (Table 1). Further, to take advantage of the vector unit of Pentium 4 , we rewrite the computationally intense part $\left(H_{w}\right.$ times a vector $Y$ ) of our program in SSE2 codes $[11,12,4]$, which yields a speed-up by a factor of $\simeq 1.8$. 
Table 1

The execution time (in unit of second) of a Pentium $4(2 \mathrm{GHz})$ node to compute 12 columns of overlap Dirac quark propagators, versus the size of the lattice. The parameters for the test are: the degree of Zolotarev rational polynomial is $n=16$, the number of bare quark masses is $N_{m}=16$ (with $m a \geq 0.02$ ), the precision of each projected eigenmode satisfies $\|\left(H_{w}^{2}-\lambda^{2}\right)|x\rangle \|<10^{-13}$, and the stopping criterion for the inner and outer CG loops is $\epsilon=10^{-11}$.

\begin{tabular}{|c|c|c|c|c|c|c|c|c|}
\hline Lattice & $\beta$ & $\begin{array}{c}\text { project. } \\
\text { no./time }\end{array}$ & $\lambda_{\min } / \lambda_{\max }$ & $\begin{array}{c}\chi \operatorname{sym} \\
\sigma(\max .) \\
\end{array}$ & $\begin{array}{l}\text { inner CG } \\
\text { ave. iters. }\end{array}$ & $\begin{array}{l}\text { outer CG } \\
\text { tot. iters. }\end{array}$ & $\begin{array}{c}\text { disk I/O } \\
\text { time }\end{array}$ & $\begin{array}{l}\text { Total } \\
\text { time }\end{array}$ \\
\hline $8^{3} \times 24$ & 5.8 & $32 / 4725$ & $0.198 / 6.207$ & $5.3 \times 10^{-14}$ & 403 & 1282 & 0 & 63550 \\
\hline $10^{3} \times 24$ & 5.8 & $30 / 7803$ & $0.152 / 6.204$ & $6.4 \times 10^{-14}$ & 519 & 1943 & 0 & 218290 \\
\hline $12^{3} \times 24$ & 5.8 & $30 / 13258$ & $0.129 / 6.211$ & $9.8 \times 10^{-14}$ & 608 & 2840 & 0 & 625718 \\
\hline $16^{3} \times 32$ & 6.0 & $20 / 74937$ & $0.215 / 6.260$ & $3.3 \times 10^{-13}$ & 370 & 3968 & 66976 & 2095975 \\
\hline
\end{tabular}

\section{Conclusions}

The speed of our system of 30 nodes is higher than 30 Gflops, and the total cost of the hardware is less than US\$30,000. This amounts to price/performance ratio better than $\$ 1.0 /$ Mflops for 64-bit (double precision) computations with overlap Dirac quarks. The basic idea of optimization is to let each node work on one of the 12 columns of the quark propagators (for a set of bare quark masses), and also use the hard disk as virtual memory for the vectors in the outer CG loop, while the $\mathrm{CPU}$ is working on the inner $\mathrm{CG}$ loop.

With Zolotarev optimal rational approximation to $\left(H_{w}^{2}\right)^{-1 / 2}$, projection of high and lowlying eigenmodes of $H_{w}^{2}$, the multi-mass CG algorithm, the SSE2 acceleration, and the memory management, we are able to compute overlap Dirac quark propagators of 16 bare quark masses on the $16^{3} \times 32$ lattice, with the precision of quark propagators up to $10^{-11}$ and the precision of exact chiral symmetry up to $10^{-12}$, at the rate of two gauge configurations ( $\beta=6.0$ ) per two days, with our present system of 30 nodes (Table 1). This demonstrates that an optimized Linux PC cluster can be a viable computational system to extract physical quantities from lattice QCD with overlap Dirac quarks [13].

\section{REFERENCES}

1. H. Neuberger, Phys. Lett. B 417, 141 (1998)
2. R. Narayanan and H. Neuberger, Nucl. Phys. B 443, 305 (1995)

3. T. W. Chiu, "A Parallel Computer For Lattice Gauge Theories," Proceedings of the Third Conference on Hypercube Concurrent Computers and Applications, edited by G.C. Fox, published by ACM, New York, N.Y.(1988) p. 81-91.

4. T. W. Chiu, T. H. Hsieh, C. H. Huang and T. R. Huang, hep-lat/0208039.

5. T. W. Chiu, Phys. Rev. D 60, 034503 (1999)

6. P. H. Ginsparg and K. G. Wilson, Phys. Rev. D 25, 2649 (1982).

7. T. W. Chiu and S. V. Zenkin, Phys. Rev. D 59, 074501 (1999)

8. E. I. Zolotarev, Zap. Imp. Akad. Nauk. St. Petersburg, 30 (1877), no. 5; reprinted in his Collected works, Vol. 2, Izdat, Akad. Nauk SSSR, Moscow, 1932, p. 1-59.

9. N. I. Akhiezer, "Theory of approximation", Dover, New York, 1992; "Elements of the theory of elliptic functions", Translations of Mathematical Monographs, 79. American Mathematical Society, Providence, R.I. 1990.

10. T. W. Chiu, T. H. Hsieh, C. H. Huang and T. R. Huang, hep-lat/0206007.

11. ftp://download.intel.com/design/perftool/ cbts/appnotes/sse2/w.fp_precision.pdf

12. M. Luscher, Nucl. Phys. Proc. Suppl. 106, 21 (2002)

13. T. W. Chiu and T. H. Hsieh, Phys. Rev. D 66, 014506 (2002); Phys. Lett. B 538, 298 (2002); hep-lat/0208066. 